Revista de Psicología Vol. 36 (1), 2018 (ISSN 0254-9247)

\title{
El encargado de convivencia escolar en Chile: hacia la comprensión de su identidad laboral ${ }^{1}$
}

\author{
Jaime Valenzuela ${ }^{2}$, Iván Ahumada ${ }^{3}$, Andrea Rubilar ${ }^{4}$, Verónica López y \\ Carolina Urbina ${ }^{6}$ \\ Centro de Investigación para la Educación Inclusiva - Pontificia Universidad \\ Católica de Valparaíso, Chile
}

Abordar la convivencia y violencia en las escuelas requiere de capacidades de gestión escolar. La Ley de Violencia Escolar en Chile de 2011 promulgó la creación obligatoria de la figura del encargado de convivencia escolar (ECE). Sin embargo, la ley no estableció un perfil definido, funciones específicas, ni horas de trabajo para el ECE, y solo paulatinamente los sostenedores han ido aumentando las horas laborales para ejercer este cargo. Ello ha generado un marco flexible de funcionamiento para el ECE, que podría tener implicancias en la construcción de su identidad laboral. Este estudio exploratorio utilizó un método de investigación cualitativa a partir del desarrollo de un estudio caso único de un ECE. Se produjo información a través de un grupo focal con el comité de convivencia escolar y entrevistas en profundidad al ECE de la escuela. Usando el método de análisis de contenido, los resultados revelan la ambigüedad de la identidad del ECE, por contar con roles segmentados en las áreas de pedagogía,

1 Esta investigación se enmarca en el Proyecto FONDECYT 1140960 "Políticas y prácticas de apoyo a la convivencia escolar en ambientes punitivos, segregados y de high-stakes testing: el caso de Chile" y CIE 160009.

2 Psicólogo por la Pontificia Universidad Católica de Valparaíso. Dirección postal: Dirección postal: Avenida El Bosque 1290, Viña del Mar, Chile. Contacto j.valenzuela.gaete@gmail. com

3 Psicólogo por la Pontificia Universidad Católica de Valparaíso. Dirección postal: Dirección postal: Avenida El Bosque 1290, Viña del Mar, Chile. Contacto: iahumadap@hotmail.com

4 Psicóloga por la Pontificia Universidad Católica de Valparaíso. Dirección postal: Dirección postal: Avenida El Bosque 1290, Viña del Mar, Chile. Contacto: andrea.rubilar.a@gmail.com

5 Doctora en Psicología. Directora del Programa de Apoyo a la Convivencia Escolar y del Centro de Investigación para la Educación Inclusiva. Profesora titular de Psicología de la Pontificia Universidad Católica de Valparaíso. Dirección postal: Avenida El Bosque 1290, Viña del Mar, Chile. Contacto: veronica.lopez@pucv.cl

6 Doctora en Psicología e investigadora del Programa de Apoyo a la Convivencia Escolar de la Pontificia Universidad Católica de Valparaíso. Dirección postal: Avenida El Bosque 1290, Viña del Mar, Chile. Contacto: carolina.urbina@pucv.cl 
administración y convivencia escolar. Se discute cómo en la práctica escolar la noción de convivencia es separada de las dimensiones de pedagogía y gestión, y cuáles son sus efectos. Palabras clave: identidad, encargado de convivencia escolar, convivencia escolar, violencia escolar, ley.

\section{School Climate Coordinators in Chile: Understanding their Labor Identity}

Addressing school climate and violence in schools requires school management skills. The 2011 School Violence Act in Chile promulgated the mandatory creation of the school climate coordinator (SCC). However, the law did not establish a defined profile, specific functions, or working hours for the SCC, and only recently have school administrators given SCCs more time for this position. This has created a flexible operating framework for the position, which could have implications in terms of the labor identity of the SCCs. This exploratory study employed a qualitative case study. An exploratory focus group was conducted with the school climate committee and three in-depth interviews with the SCC of one municipal school. Content analyses revealed the ambiguity of the SCC figure due to a fragmentation of roles in the areas of pedagogy, administration and school climate, creating a scenario where the roles of teacher, inspector, and SCC are segmented. We discuss how the notion of school climate is separated within the school practice from the dimensions of pedagogy school management, and its possible effects.

Keywords: identity, school climate coordinators, school climate, school violence, law

\section{El coordenador de convivência escolar em Chile: compreensão de identidade de trabalho} Abordar a convivência e a violência nas escolas requer habilidades de gestáo escolar. A Lei de Violência Escolar de 2011 no Chile promulgou a criação obrigatória do coordenador de convivência escolar (CCE). No entanto, a lei não estabeleceu um perfil definido, funçóes específicas ou horas de trabalho para a CCE, e gradualmente a equipe de suporte estava aumentando o horário de trabalho para essa posição. Isso criou um quadro operacional flexível para a CCE, que poderia ter implicaçóes para a construção de sua identidade de trabalho. Este estudo exploratório utilizou um método de pesquisa qualitativa a partir do desenvolvimento de um único estudo de caso de um CCE. A informaçáo foi produzida através de um grupo focal com o comitê de convivência escolar e entrevistas em profundidade com a CCE da escola. Usando o método de análise de conteúdo, os resultados revelam a ambiguidade da identidade da ECE, porque tem papéis segmentados nas áreas de pedagogia, administração e convivência escolar. É discutido como na prática escolar a noção de convivência é separada das dimensóes da pedagogia e gestão, e quais são seus efeitos.

Palavras-chave: identidade, coordenadores de convivência escolar, convivência escolar, violência escolar, lei.

\section{La personne en charge de la coexistence scolaire au Chili: vers la compréhension de son identité de travail}

L'approche de la coexistence et de la violence dans les écoles nécessite des compétences en gestion scolaire. La loi sur la violence scolaire au Chili de 2011 a promulgué la création obligatoire de la personne en charge de la coexistence scolaire (ECE). Cependant, la loi n'a pas établi de profil défini, de fonctions spécifiques ou d'heures de travail pour la ECE, et ce n'est que progressivement que les partisans ont augmenté leurs heures de travail pour exercer ce poste. Cela a généré un cadre opérationnel souple pour la ECE, ce qui pourrait avoir 
El encargado de convivencia escolar en Chile / Valenzuela et al.

des implications pour la construction de leur identité de travail. Cette étude exploratoire a utilisé une méthode de recherche qualitative à partir du développement d'une seule étude de cas d'une ECE. L'information a été produite à travers un groupe de discussion avec le comité de coexistence de l'école et des entretiens approfondis avec l'ECE de l'école. À l'aide de la méthode d'analyse de contenu, les résultats révèlent l'ambiguïté de l'identité de la ECE, pour avoir des rôles segmentés dans les domaines de la pédagogie, de l'administration et de la coexistence scolaire. Il est discuté comment dans la pratique scolaire la notion de coexistence est séparée des notions de pédagogie et de gestion, et quels sont leurs effets.

Mots clés: identité, personne en charge de la coexistence scolaire, coexistence scolaire, violence scolaire, loi.

Entendiendo la relevancia de la convivencia en los establecimientos educativos, organismos como la Unesco, PNUD y la Unicef han promovido diversos esfuerzos para su desarrollo. Así, por ejemplo, en 1990 el movimiento de Educación para Todos (EPT) presenta a la convivencia en las escuelas como una preocupación explícita, alcanzando un estatus equivalente a otros aprendizajes que se consideran indispensables en las instituciones educativas (Palomino, 2012). A partir de este escenario se han implementado una serie de políticas de convivencia escolar en Sudamérica. Por ejemplo, la ley 29719 en Perú, promueve la convivencia sin violencia en las instituciones educativas, estableciendo que convivir democráticamente en la escuela significa desenvolverse en un ambiente tolerante, solidario y promotor de autonomía (Minedu, 2011). Por su parte, Bolivia durante el año 2012 implementó el decreto supremo 1302, donde se entiende la convivencia escolar como la interrelación de los diferentes integrantes de un establecimiento, constituyendo una construcción colectiva y de responsabilidad de todos los/as componentes de la comunidad educativa (Fernández \& Fernández, 2012).

En Chile, el Ministerio de Educación propuso en el ańo 2001 la primera versión de una política de convivencia escolar, buscando generar las condiciones para el desarrollo de los estudiantes en un contexto pacífico, democrático y armónico (Mineduc, 2011). Esta política fue reeditada en 2005 y 2008, y posteriormente actualizada en el año 2015. Junto con ello, surge en 2010 el proyecto de Ley sobre Violencia Escolar (LSVE). 
Luego de una serie de modificaciones al proyecto original, esta ley se instala discursivamente como un consenso político (Carrasco, López \& Estay, 2012) que entiende la buena convivencia como "la coexistencia pacífica de los miembros de la comunidad educativa, que supone una interrelación positiva entre ellos y permite el adecuado cumplimiento de los objetivos educativos" (ley 20.536, artículo 16a). Esta definición, junto con las nuevas estructuras y consecuencias que se describen a continuación, se incorporaron en la Ley General de Educación (ley 20.370).

Entre las modificaciones incorporadas a la Ley General de Educación, y con el objetivo de establecer sanciones ante posibles casos de acoso escolar, la ley plantea que todos los colegios deben contar con un reglamento interno que indique las faltas a la convivencia y las sanciones correspondientes. También crea el Comité de Buena Convivencia Escolar, que viene a constituir un apoyo para promover una convivencia positiva en las instituciones privadas que no están legalmente obligadas a tener un consejo escolar. Adicionalmente, y para el caso de todos los establecimientos que reciben financiamiento del Estado - que en Chile son los establecimientos municipales y particular-subvencionados - y por tanto obligatoriamente deben contar con un consejo escolar, dicha ley creó la figura del encargado de convivencia escolar, con el propósito de establecer un actor que pudiera guiar la construcción del plan de gestión de la convivencia en la escuela:

Todos los establecimientos educacionales deberán contar con un encargado de convivencia escolar, que será responsable de la implementación de las medidas que determinen el Consejo Escolar o el Comité de Buena Convivencia Escolar, según corresponda, y que deberán constar en un plan de gestión (Ley 20.536, Art. 15).

Esta figura del ECE no estaba contemplada en los inicios de la discusión parlamentaria, pues fue rechazada en primera instancia en favor de la presencia exclusiva del comité de convivencia. Sin embargo, posteriormente fue aprobada bajo el argumento de que esto lograría una mejora en la gestión y en el trabajo práctico de la escuela (Chadwick, Allamand \& Cantero, 2010). No obstante, nada dice la ley respecto 
del perfil del ECE — por ejemplo, de la formación previa a nivel de pregrado o posterior- de sus funciones específicas, ni del mínimo de horas de trabajo que debe dedicar a la implementación de las medidas que determinen el Consejo Escolar o el Comité de Convivencia escolar. Tampoco la ley se refiere a la construcción, implementación y evaluación del plan de gestión.

En definitiva, la ley 20.536 crea un cargo nuevo que se agrega la estructura organizacional de los establecimientos escolares, pero deja un campo de acción amplio e indefinido. Dada la disyuntiva que plantea la existencia del encargado de convivencia escolar respecto al logro de los objetivos educativos antes mencionados, supeditado a la amenaza frente a la posible sanción que por ley ahora pueden recibir los establecimientos si no cumplen con asegurar los debidos resguardos y protocolos de convivencia escolar, es necesario evidenciar cómo viven y perciben los ECE su rol. En este artículo, consideramos que las perspectivas del rol profesional y de la identidad laboral constituyen un camino para explorar las atribuciones que los otros actores escolares tienen sobre este cargo en la estructura organizacional de las escuelas. A su vez, esta aproximación permitiría contribuir a reformular de forma consecuente las funciones de figuras individuales o colectivas que se encarguen de gestionar la convivencia

\section{El encargado de convivencia escolar (ECE)}

En el caso de Perú, la ley establece que es un equipo responsable - "el equipo de convivencia escolar" - quien tiene las funciones de planificar, implementar y evaluar el plan de convivencia democrática con la participación de las organizaciones estudiantiles (Minedu, 2012). En Bolivia, por otro lado, la prevención e intervención para enfrentar la violencia, maltrato y abuso en el ámbito escolar deviene en la creación de Comités de la Promoción de la Convivencia Pacífica (Minedubo, 2012).

En Chile, en cambio, el comité de buena convivencia queda delimitado al caso de establecimientos particulares que no tienen - por ley_ que contar con un consejo escolar. Para el caso de los 
establecimientos que reciben financiamiento del estado (escuelas municipales y particular-subvencionados), se mantiene el consejo escolar y se crea el cargo de ECE como figura individual para abordar la convivencia escolar.

Por otra parte, el hecho de que la figura del ECE se haya creado en el marco de una ley que, por primera vez, permite sancionar a los establecimientos que no cumplen satisfactoriamente con prevenir la violencia escolar y garantizar una convivencia escolar pacífica, por la vía de multas monetarias, crea una tensión para el ECE. Como señalan Magendzo, Toledo y Gutiérrez (2013) y Carrasco et al. (2012), se observan dos paradigmas antagónicos en la LSVE, uno de control y sanción, y otro formativo. Esta tensión paradigmática permitiría que bajo el paradigma del control y la sanción, y particularmente bajo una visión jerárquica de la convivencia, suceda que:

El Comité de Buena Convivencia Escolar, así como el encargado responsable de buena convivencia, pondrán el acento en maximizar la vigilancia y el control [...] advertirán constantemente de las posibles consecuencias que tiene contravenir las normas y, por sobre todo, estarán muy preocupados por completar los formularios que las autoridades elaboren y demanden (Magendzo et al., 2013, p. 387).

Así, considerando las investigaciones internacionales y el paradigma de la convivencia democrática, se esperaría que la labor del ECE favorezca el fortalecimiento de las estructuras de toma de decisiones con representación de la comunidad educativa (Carrasco, López \& Estay, 2012). No obstante, dado el escenario de paradigmas en tensión de la ley previamente identificados por dos estudios independientes entre sí (Magendzo et al., 2013; Carrasco et al., 2012), los paradigmas de la ley abren un campo donde el ECE podría trabajar tomando decisiones de forma autónoma y/o dando cuenta a autoridades específicas como el director o la jefatura de la Unidad Técnico-Pedagógica (UTP), o por el contrario, podría promover más bien un trabajo en equipo, donde se compartan y discutan opiniones con el consejo escolar o el comité de la buena convivencia. 


\section{La identidad laboral del ECE}

La LSVE no define las funciones del ECE, tampoco asigna horas de trabajo ni determina el perfil profesional del mismo, bajo el entendido de que estos aspectos forman parte de las decisiones que debe adoptar cada establecimiento y/o sostenedor (Mineduc, 2013), en un contexto de política educativa que favorece la desregulación del Estado. Como una forma de abordar estos aspectos que dan cuenta de una labor aún por definirse en la escuela, consideramos relevante indagar en la identidad laboral del ECE que se construiría bajo esta lógica amplia de funcionamiento. Para ello, hemos considerado como marco de referencia teórica el constructo de identidad abordado por Gleizer (ver Garabito, 2005), el cual refiere a una forma de acción del individuo sobre sí mismo. Esta forma de acción implica, además de reflexión, un proceso de identificación, una acción sobre el mundo social y el mundo de vida.

La identidad laboral del ECE implica su concepción de trabajador, pero además, su relación con los demás actores educativos, que configura al fin y al cabo un conjunto de acciones que construyen continuamente la convivencia en la escuela. Garabito (2005) ha definido tres dimensiones centrales de la identidad laboral, a través de las cuales el sujeto establece concepciones sobre el trabajo que realiza.

La primera dimensión es el mundo intersubjetivo. Este se entiende como un diálogo constante en el que se da cabida a interpretaciones que el individuo hace de su relación con otros. En el mundo intersubjetivo se encuentran las emociones, valoraciones y creencias del sujeto, dando paso a un constante construir y reconstruir (Garabito, 2005). En este plano, se podría observar como el ECE percibe su relación con otros actores educativos, particularmente en lo que respecta cotidianamente a su labor.

La segunda dimensión es el mundo social, que involucra la percepción del sujeto sobre las estructuras sociales y económicas que dictan las formas de actuar. Bajo esta dimensión, se entiende que el trabajo existe como actividad social y económica que regula la vida de los individuos, 
estableciendo diversas estructuras normativas que se deben aceptar para poder trabajar (Garabito, 2005). Así, se puede visualizar un diálogo entre el poder y la dominación que se traduce en la conformación de determinadas estructuras, colectividades o grupos. En el caso del ECE, refiere al funcionamiento de cada establecimiento educacional, particularmente respecto a lo que cada institución considera necesario o urgente de cumplir.

La tercera dimensión considerada es el universo simbólico, que corresponde a las valoraciones culturales que los sujetos atribuyen al mundo social y al mundo intersubjetivo (Garabito, 2005). Esto se relaciona con el quehacer de lo que culturalmente se considera correcto y productivo, como por ejemplo, los ideales culturales sobre la inteligencia, la ética y la lealtad. En este caso se observan las valoraciones que hace el ECE respecto a los aspectos positivos y negativos de su cargo, así como la definición de estos en una esfera simbólica.

Considerando estas dimensiones, se puede entender que LSVE se constituye como un aspecto propio del universo simbólico, que se representa mediante la estructura normativa de la escuela y se pone en práctica en el mundo intersubjetivo de cada actor educativo. Sin embargo, como la ley no designa funciones específicas al ECE, salvo la planificación y ejecución del plan de gestión, la identidad del ECE se perfila como algo por definir, por lo que es pertinente preguntarse: ¿Cómo se construye la identidad laboral de un ECE? Como establece el propio Ministerio de Educación de Chile (Mineduc, 2011), mucho de lo que sucede en la escuela no está escrito y puede realizarse sin la reflexión necesaria, por lo que se hace necesario identificar conocimientos, habilidades y actitudes para intencionar su coherencia con los aprendizajes en convivencia.

Por lo anterior, en este estudio buscamos comprender cómo construye la identidad laboral un ECE de la región de Valparaíso. Esto se realizó a partir de la descripción e interrelación de cada una de las dimensiones anteriores, con el fin de observar, documentar y analizar cómo este actor educativo intenta resolver aquellas tensiones de la ley para desarrollar un marco de acción para gestionar la convivencia escolar. 
El encargado de convivencia escolar en Chile / Valenzuela et al.

\section{Método}

El estudio se enmarcó en un paradigma fenomenológico con una metodología de tipo cualitativa (Cáceres, 2003). La elección del caso se realizó a partir de un muestreo por conveniencia, procedimiento que consiste en la selección de unidades de muestra a partir del criterio de los investigadores, y eligiendo las unidades de acuerdo a su fácil disponibilidad (Mejía, 2000). Este muestreo, según Collazos (2007), se define como la caracteristica de aquellas investigaciones cuyo tema elegido ha sido poco explorado y reconocido, lo cual es coherente con el cargo de creación reciente sobre el que no existen estudios previos. En consecuencia, la selección del establecimiento se realizó considerando la opinión experta de los actores claves de la Superintendencia de Educación y de la Unidad de Convivencia Escolar de una Corporación Municipal de la Región de Valparaíso, dado que estos poseían experiencia y conocimiento específico sobre la posición del ECE dentro de una población de posibles participantes.

\section{Participantes}

El muestreo por conveniencia permitió contar con la participación de un ECE que trabajaba en una escuela municipal de la región de Valparaíso, y que tenía la particularidad de poseer un Comité de la Buena Convivencia. La escuela imparte solo enseñanza básica y tiene un alto índice de vulnerabilidad escolar (82\%), lo que significa que un alto porcentaje de sus estudiantes provienen de contextos socioeconómicos medio-bajos. Según datos oficiales del Gobierno de Chile, ambos son factores que inciden no con la cantidad, sino con el tipo de violencia que se ejerce, siendo esta de un corte más físico que psicológico (Gobierno de Chile, 2006a).

El participante del estudio es un ECE que posee una variada experiencia laboral, ya que previamente se desempeńaba como profesor de inglés y actualmente es tanto inspector general como ECE del mismo establecimiento. Tal situación parece relevante para establecer cómo esta experiencia laboral previa se conjuga con la labor actual del ECE. 


\section{Técnicas de producción de información}

En un primer momento se realizó un grupo focal como manera preliminar de recabar información relativa a la participación del ECE en la escuela. Como indica Korman (2003), el grupo focal es una reunión de un grupo de individuos seleccionados por los investigadores para discutir y elaborar, desde la experiencia personal, una temática o hecho social que es objeto de investigación (Mella, 2000). En este caso, los participantes fueron los integrantes del equipo de convivencia escolar (asistente social, psicólogo primer ciclo, psicólogo segundo ciclo, docente primer ciclo, docente segundo ciclo y ECE), ya que se sostuvo como criterio aquellos que mantenían una cercanía y comunicación constante con la figura del ECE (ver Tabla 1).

\section{Tabla 1}

Participantes de las técnicas de producción

\begin{tabular}{ll}
\hline Participantes & Técnica \\
\hline Integrantes del equipo de convivencia: & \\
Encargado de convivencia escolar & \\
2 representantes consejo de profesores & Grupo focal \\
Psicólogo & \\
Psicóloga & \\
Trabajador social & 3 entrevistas en profundidad \\
\hline Encargado de convivencia escolar &
\end{tabular}

Luego, se realizaron tres entrevistas en profundidad con el ECE. Como señalan Deslauriers y López (2011), las entrevistas en profundidad permiten obtener detalles del discurso y eliminar dificultades con el objetivo de acceder al ámbito privado del personaje en cuestión. Aquellas tuvieron la intención de profundizar en aspectos propios de las tres dimensiones de la identidad laboral (Garabito, 2005), particularmente del mundo intersubjetivo que no apareció suficientemente representado en la instancia grupal. Cabe destacar el carácter recursivo de la producción de información, ya que cada instancia fue analizada 
para dar paso a la construcción de la siguiente. Esto se hizo pensando en establecer una revisión constante que permitiera ir retroalimentando la información. Lo anterior fue particularmente relevante en la tercera entrevista, donde se le presentó con anterioridad al ECE un documento con la categorización preliminar de los datos para que él pudiera establecer su posicionamiento respecto a las mismas (ver Tabla 1).

\section{Técnicas de análisis de la información}

Se realizó un análisis de contenido cualitativo de la información. Este procedimiento permite un análisis de comunicaciones de carácter comprensible y alcanzable, pero que al mismo tiempo conserva el grado de rigor en la sistematización concebida en un principio (Cáceres, 2003). Para realizar este análisis, se utilizó el programa de análisis cualitativo de datos Atlas ti versión 5.0. El primer momento de análisis consistió en una categorización emergente que permitió un levantamiento de referenciales significativos a partir de la propia indagación (Cisterna, 2005). En un segundo momento, se definieron códigos y categorías de clasificación derivados de la información producida en las entrevistas y grupo focal. Estos fueron relacionados, en un tercer momento, con las categorías a priori, es decir, aquellas construidas antes del proceso recopilatorio de información y provenientes del marco de referencias teórica (Cisterna, 2005), y que corresponden a las tres dimensiones de la identidad laboral de Garabito (2005), a saber: a) mundo intersubjetivo, donde se encuentran las interpretaciones que el participante tiene de su trabajo como ECE, donde se expresan creencias, valores y emociones; b) mundo social, en el cual se presentan las percepciones que el ECE tiene de la institución educativa y su rol como trabajador; y c) universo simbólico, que aborda las valoraciones de la comunidad educativa respecto al trabajo del ECE. Por lo tanto, el procedimiento de análisis implicó una primera fase emergente e inductiva, que luego fue puesta en relación con categorías teóricas definidas previamente. 


\section{Procedimiento}

A partir del diálogo consensuado con la coordinadora de la Corporación Municipal, principalmente en base a la disponibilidad de los establecimientos y de los encargados de convivencia, se efectuó el contacto con el director de la escuela y el inspector general de la misma, que además se desempeńa como ECE. Habiendo recibido la autorización por parte de estos agentes educativos, se procedió a firmar el consentimiento informado y a explicar los alcances y requerimientos de la investigación. El grupo focal y las entrevistas fueron realizados en el establecimiento escolar, durante la jornada escolar.

En torno a las consideraciones éticas, se solicitó la autorización de la autoridad correspondiente al establecimiento en el cual se desarrolló la investigación. Todos los participantes firmaron un consentimiento informado, en el que se les informó los objetivos del estudio y se formalizó su compromiso de participación libre y voluntaria, junto con el resguardo de la confidencialidad de todos los datos y acciones que surjan en su producción. Junto con lo anterior, se tuvo presente la obligación ética de involucrar a los participantes a través de la negociación constante y la devolución final de la información producida. Considerando que esto puede ser una posibilidad de construir relato e interpretación desde un punto de vista colaborativo y democrático, la devolución supuso un punto de encuentro para la cooperación, para crear vínculos y compromisos con los sujetos, que se implican como autores en la interpretación de su propia vida (Rivas \& Leite, 2010).

\section{Resultados}

El análisis de contenido permitió crear 22 códigos y 6 Familias (F) de clasificación. Estos elementos fueron relacionados con los tres niveles de la identidad laboral definidos con anterioridad, y que forman parte de los objetivos específicos de la investigación (Tabla 2). 
El encargado de convivencia escolar en Chile / Valenzuela et al.

\section{Tabla 2}

\section{Códigos y categorías}

\begin{tabular}{|c|c|c|c|}
\hline $\begin{array}{l}\text { Niveles } \\
\text { de ident. } \\
\text { laboral }\end{array}$ & $\begin{array}{l}\text { Criterios de } \\
\text { análisis }\end{array}$ & Familias & Códigos \\
\hline 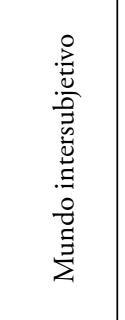 & $\begin{array}{l}\text {-Trayectoria } \\
\text { laboral del } \\
\text { sujeto } \\
\text {-Funciones } \\
\text { desempeńadas } \\
\text {-Dificultades } \\
\text { relacionadas a } \\
\text { su labor }\end{array}$ & $\begin{array}{l}\text { F1 } \\
\text { Experiencia del } \\
\text { ECE en torno } \\
\text { a su cargo }\end{array}$ & $\begin{array}{l}\text { (A) Percepción de funciones que intentan unir lo } \\
\text { normativo y lo formativo } \\
\text { (B) Percepción de elementos pocos definidos en } \\
\text { la labor de ECE } \\
\text { (C)Distinción entre las labores realizadas como } \\
\text { ECE e inspector } \\
\text { (D) Percepción de la dificultad de ser ECE e ins- } \\
\text { pector en el mismo horario } \\
\text { (E) Percepción de las diferentes labores reali- } \\
\text { zadas a lo largo de la trayectoria laboral }\end{array}$ \\
\hline \multirow{3}{*}{ 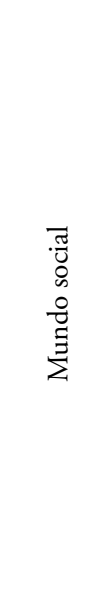 } & \multirow{3}{*}{$\begin{array}{l}\text {-Contexto } \\
\text { escolar } \\
\text {-Jerarquías y } \\
\text { normas del } \\
\text { establecimiento } \\
\text {-Conformación } \\
\text { de grupos } \\
\text {-Tiempo } \\
\text { cotidiano }\end{array}$} & $\begin{array}{l}\text { F2 } \\
\text { Posibilidades } \\
\text { para la reso- } \\
\text { lución de } \\
\text { conflictos } \\
\text { normativos }\end{array}$ & $\begin{array}{l}\text { (F) Posibilidad de crear cargos administrativos } \\
\text { (G) Posibilidad de delimitar la concepción de } \\
\text { convivencia } \\
\text { (H) Posibilidad de delimitar las funciones del } \\
\text { cargo } \\
\text { (I) Posibilidad de protocolizar las funciones }\end{array}$ \\
\hline & & $\begin{array}{l}\text { F3 } \\
\text { Nociones } \\
\text { caracterizadas } \\
\text { como clave en } \\
\text { la convivencia }\end{array}$ & $\begin{array}{l}\text { (J) Nociones sobre el papel que toma la gestión } \\
\text { de la convivencia en la vida escolar } \\
\text { (K) Nociones sobre el papel que toman las acti- } \\
\text { vidades formativas en la convivencia } \\
\text { (L) Nociones sobre del papel que toman las } \\
\text { normas en la escuela }\end{array}$ \\
\hline & & $\begin{array}{l}\text { F4 } \\
\text { Elementos que } \\
\text { dificultarían la } \\
\text { realización del } \\
\text { cargo de ECE }\end{array}$ & $\begin{array}{l}\text { (M) Visualización de actividades adicionales al } \\
\text { propio cargo } \\
\text { (N) Descripción de problemas relativos a la eje- } \\
\text { cución de lo administrativo } \\
\text { (O) Descripción de situaciones asociadas al } \\
\text { ambiente de vulnerabilidad que habría en la escuela }\end{array}$ \\
\hline \multirow{2}{*}{ 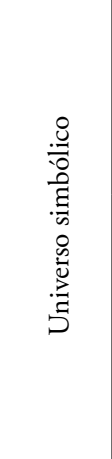 } & \multirow[t]{2}{*}{$\begin{array}{l}\text {-Valoraciones } \\
\text { culturales del } \\
\text { trabajo }\end{array}$} & $\begin{array}{l}\text { F5 } \\
\text { Diferenciación } \\
\text { entre lo } \\
\text { administrativo, } \\
\text { pedagógico y } \\
\text { la convivencia } \\
\end{array}$ & $\begin{array}{l}\text { (P) Comparación entre las labores administra- } \\
\text { tivas, pedagógicas y convivencia } \\
\text { (Q) Diferenciación entre las labores pedagógicas } \\
\text { y de convivencia } \\
\text { (R) Diferenciación entre las labores administra- } \\
\text { tivas y de convivencia }\end{array}$ \\
\hline & & $\begin{array}{l}\text { F6 } \\
\text { Descripción } \\
\text { de elementos } \\
\text { ideales para el } \\
\text { cargo de ECE }\end{array}$ & $\begin{array}{l}\text { (S) Indicaciones sobre la relevancia de las com- } \\
\text { petencias blandas en el cargo de ECE } \\
\text { (T) Indicaciones sobre la relevancia de conocer } \\
\text { las dinámicas de la escuela } \\
\text { (U)Indicaciones sobre la relevancia de tener } \\
\text { experiencia en aula } \\
\text { (V)Indicaciones sobre la relevancia de establecer } \\
\text { un trabajo colaborativo entre actores }\end{array}$ \\
\hline
\end{tabular}




\section{El mundo intersubjetivo del ECE}

Destaca el intento del participante de dar sentido tanto a las labores de inspector como de ECE. Como menciona De la Garza (citado en Garabito, 2005), los sujetos dan sentido y deciden sus cursos de acción. En este caso, el participante para dar sentido busca unificar los aspectos punitivos y formativos de los cargos de ECE e inspector, al destacar la importancia de equilibrar ambas posiciones en la misma persona.

Creo que igual es importante que la persona que encabece la convivencia escolar también esté a cargo de las normas y las regulaciones, porque si no yo solamente sería como un juez de la república (Segunda entrevista, ECE).

Sin embargo, la incompatibilidad horaria, descrito como el elemento que divide ambos cargos en lo funcional, se presenta como algo que dificulta la opción de congeniar las posiciones:

En este último tiempo yo siento que no podía planificar la convivencia escolar, porque no me daba el tiempo (ECE, Segunda entrevista).

Entendiendo que el trabajo es un escenario donde confluyen diferentes subjetividades, Friedmann (citado en Garabito, 2005) postula, entre ellas, la vivida en el quehacer cotidiano del trabajo, que va desde los estados de insatisfacción a la alegría. Se destaca que para este ECEinspector existe cierta insatisfacción por la organización de sus horarios que le impide participar de manera más activa en la gestión de la convivencia escolar en la escuela. Es por esto que el participante realiza una distinción entre las labores realizadas como inspector y ECE, temática que se relaciona con el intervalo temporal de carácter objetivo que posee el mundo intersubjetivo, en específico; la duración de un contrato (Garabito, 2005). Dicho contrato en el cargo del ECE no existe, ya que la ley deja a los establecimientos la responsabilidad de elegir qué funciones debe cumplir el encargado y en qué tiempos, aportando así a la percepción de que hay elementos poco definidos en la labor de ECE.

En otro plano, las dificultades no solamente existen en el ámbito de la organización de los horarios, sino también de conocimiento, ya 
que en Chile la ley tiende a plantear que son quienes trabajan de forma remunerada en cada colegio, los que reciben capacitaciones sobre la promoción de la buena convivencia escolar (Carrasco et al., 2012). Es así que el ECE trabaja sin haber recibido capacitación más que su experiencia laboral como docente de inglés, inspector general y finalmente como ECE. Para poder solventar estas situaciones, aparece un tópico que es propio del mundo intersubjetivo, esto es, la relación establecida con otros, ya que el ECE destaca lo relevante que es la colaboración con el equipo de convivencia. Esto no es solo descrito en términos laborales sino también de formación, ya que la relación con los psicólogos y el asistente social le ha permitido, según relata, profundizar en las concepciones de convivencia.

Es así que, si bien el ECE seguiría ocupando la mayor parte de su tiempo en el cargo de inspector, en el plano de las concepciones ha podido establecer una mayor unidad entre los aspectos propios de lo punitivo y formativo gracias al apoyo que recibe del equipo de convivencia. Esto se relaciona con lo que plantea el Mineduc (2013) cuando indica que la convivencia implica una mirada integradora y transdisciplinaria, que requiere de la participación de todos los actores de la comunidad educativa.

\section{El mundo social del ECE}

Se puede establecer que el problema con los horarios y la visualización de actividades adicionales al propio cargo se relacionan con elementos estructurales que sobrepasan la propia acción cotidiana del individuo. Ante el marco de acción flexible que establece la LSVE, cabe preguntarse ¿De quién es la responsabilidad de delimitar dichas funciones y retribuciones a la labor del ECE?

El mundo social es el encargado de organizar tanto el tiempo cotidiano como el tiempo de vida del individuo (Garabito, 2005). De acuerdo al tiempo cotidiano se debe determinar en un corto plazo la jornada laboral del trabajador, y en función de aquello, los tiempos de estudio y/o descanso que se le atribuyen. En lo que respecta al tiempo de vida, el trabajo se caracteriza por ser un episodio trascendente, que 
determina la biografía personal, y se erige como elemento central del proyecto de vida individual (Garabito, 2005). Es por esto que, de acuerdo al contexto de nuestra investigación, sería la institucionalidad educativa, como estructura jerárquica, social y económica, la que debiera establecer los parámetros satisfactorios, para la labor y funcionamiento del ECE.

(...) tiene que estar definido, sino no tienes cómo evaluar; si no tengo claro el objeto de la función que cumple ¿Cómo voy a poder evaluar tu trabajo? (...) entonces la comunidad va a esperar cualquier cosa del $E C E$. (Segunda entrevista, ECE)

La cita anterior da cuenta de la falta y luego la necesidad de delimitación del cargo. En este caso, se denota que son las labores administrativas las que explican estas dificultades horarias, ya que el ECE, bajo el cargo de inspector, no solo se dedicaría a las labores de mantención de la escuela, puesto que apoya además a los otros actores en la realización de sus protocolos para evitar multas:

Hay más inspectores, pero el riesgo de que comentan un error es muy alto, por ejemplo, me pasaba que cuando revisaba el libro siempre encontraba errores, entonces esos errores cuestan dinero (...) (Segunda entrevista, ECE).

Este escenario da cuenta de las autoridades que toman medidas, pero a su vez se encuentran vigiladas arriesgando sanciones normalizadoras de su rol (Carrasco et al., 2012), lo que implica que la Ley sobre Violencia Escolar propone la creación de un nuevo cargo para abordar la convivencia (ECE), pero sin hacer mayores cambios a la estructura normativa donde existe la vigilancia y control.

Aunque el discurso del ECE denota posibles soluciones a esta situación, como son la creación de un cargo administrativo, la delimitación del cargo y la protocolarización de funciones, en distintos momentos de las entrevistas aparecen como más relevantes aquellas propuestas del ECE que enfatizan en los aspectos formativos de la convivencia. Las primeras ideas implican realizar modificaciones que no se harán en el corto plazo, y por tanto significan reproducir un sistema que le es impuesto. 
En contraste a ello, las actividades formativas, aunque no siempre son las más valoradas, nos hablan de la relevancia de la gestión de la convivencia en los establecimientos, en tanto producen interacciones sociales y vinculaciones distintas a las que se producen al interior del aula, y que pueden transformarse en una buena oportunidad de poner en práctica los aprendizajes en convivencia (Mineduc, 2013).

\section{El universo simbólico del ECE}

El universo simbólico, que se considera símbolo de lo correcto, de lo bueno y lo productivo (Garabito, 2005), pone en juego aquellas valoraciones culturales que establecen lo que es más deseable para la persona que cumple el rol de ECE. Ante una definición ambigua del espectro de acción del ECE, cabe preguntarse ¿Bajo qué premisas el ECE puede tener una labor satisfactoria? En lo que respecta al ECE de esta investigación, se entiende que el cargo conlleva un gran peso subjetivo para el individuo, ya que la sociedad no solo demanda trabajar para lograr integrar la actividad económica, sino que, para llegar a ser un hombre de bien, también debe trabajar correcta y productivamente (Garabito, 2005).

De ahi que cuando yo asumi estás horitas fue súper bueno porque fui elaborando lo que yo pensaba que podía ser la convivencia escolar, de ahi que creo que fue positivo, y debería hacerse una inducción más profunda respecto al rol (Segunda entrevista, ECE).

En la cita anterior, vemos que el participante trata de dar cuenta de lo que es el cargo, a partir de sus valoraciones personales, considerando la importancia del aprendizaje, pero al mismo tiempo demandando una inducción respecto al rol. Esto último no es extraño, considerando que podría estar mencionando lo positivo desde un ambiente donde la implementación de mecanismos de apoyo se ve dificultada ya que las instituciones educativas no siempre cuentan con recursos materiales y humanos preparados para ello, ni con la voluntad tan clara de los sostenedores para hacerla funcionar (Carrasco et al., 2012). 
En este plano, cuando el ECE no recibe orientaciones que le permitan guiarse en su labor, realiza valoraciones culturales respecto a un perfil ideal del cargo. Estas valoraciones no dejan de relevar ciertas contradicciones, puesto que se destaca la importancia de las competencias blandas como el respeto, el buen trato y el trabajo en equipo, aunque estableciendo al mismo tiempo esto mismo como un factor de exclusión a ciertos actores educativos en lugar de apoyarlos:

... hay gente que no sabe trabajar en equipo, y esas personas no sirven para el cargo de ECE (Tercera entrevista, ECE).

Esto sumado a otro tipo de exclusiones referentes a la falta de conocimiento en aula, la experiencia en la escuela, va a la par con la diferenciación entre lo administrativo, la pedagogía y la convivencia. En variadas ocasiones a lo largo de las entrevistas, los participantes dan cuenta de una escisión entre el eje del aprendizaje y de la convivencia, como si se tratasen de dos asuntos con racionalidades distintas (Carrasco et al., 2012). En ese plano, el ECE postula, de forma contradictoria, que si bien las áreas están divididas se pueden comunicar, contraponiéndose a la incompatibilidad horaria descrita previamente que dificulta la coordinación efectiva entre actores educativos.

\section{La identidad laboral en el marco de la dualidad ECE-inspector}

En relación a la dualidad dada en este caso de estudio entre los roles de inspector y de ECE, se observa que si bien las escuelas visualizan la creación del cargo de ECE para abarcar la convivencia, se sigue manteniendo al inspector en su rol original: el rol de normalización y sanción. Como establece el mismo nombre de la ley (Ley sobre Violencia Escolar), la convivencia escolar aparece como algo que se promueve, y la violencia escolar, como algo que se previene, definiendo ciertas categorías de relevancia al interior de las formas de manifestación de la violencia en el espacio escolar (Carrasco et al., 2012).

En el caso del participante de este estudio, esto se resuelve generando una ambigüedad identitaria en lo intersubjetivo, ya que el interés por formar estudiantes más solidarios, respetuosos, socialmente- 
responsables y con capacidad para incidir en la vida democrática del país (Mineduc, 2013) se pone en paralelo con las medidas para prevenir y proteger a los estudiantes de todo tipo de riesgos, entre ellos, el acoso $\mathrm{u}$ hostigamiento permanente (bullying), agresiones sexuales y delitos de connotación sexual y el consumo de drogas (Mineduc, 2013). Esto hace necesario una relación más cohesionada entre el ECE y el comité de convivencia para dar apoyo respecto a actividades y formación en convivencia escolar, en conjunto con la socialización de la figura de ECE como entidad capaz de trabajar en equipo. Después de todo, el ECE es un actor clave que necesita de la coordinación con otros, en la medida de que establece mecanismos y estrategias para instalar sistemas de trabajo colaborativos e interdisciplinarios (Mineduc, 2013), ya que slo así se puede conjugar la posición jerárquica e individual que clásicamente se visualiza en el inspector, con la formatividad de la convivencia escolar.

Desde ese punto de vista, al entender la posición del inspector en las escuelas como una figura que vigila y sanciona, también es necesario plantearse hasta qué punto debe tener una visión distinta o incluso ser un cargo distinto del de ECE, ya que ambos cargos debieran apuntar a potenciar la organización colectiva entre padres, profesores y estudiantes alrededor de un Plan de Gestión, aprovechando para ello la propia definición que la ley otorga respecto de convivencia escolar (Carrasco et al., 2012).

Al parecer, la Ley sobre Violencia Escolar no profundizó suficientemente en este aspecto, ya que decidió crear una nueva figura de ECE sin replantearse las funciones que desempeńan los actores ya existentes, y sin revisar cuál sería el impacto en términos identitarios de tener a una misma persona con dos roles institucionales. Se entiende que las labores del ECE, al no estar reguladas más allá de la coordinación, elaboración e implementación del plan de gestión de la convivencia (Mineduc, 2013), ya no serían establecidas por autoridades externas sino por los propios actores del establecimiento, lo que lleva a preguntarse: ¿Quién o quiénes establecen el margen de acción del ECE en la escuela? 
En primer lugar, haremos referencia al discurso del entrevistado, ya que, al diferenciar entre lo propio de la pedagogía y lo de la convivencia, él separa tajantemente las labores técnico-pedagógicas de las labores de convivencia escolar:

Si un niño dice que el profesor le puso un 2, ese es un tema pedagógico que ve la jefa de UTP (...), si yo me entrometiera en eso, estaría pasando a llevar su rol. Pero si ella dice que el niño tiene puros 2 porque algo más está pasando; se transforma en un problema de convivencia (Segunda entrevista, ECE).

Esto da a entender que cuando existe un problema que afecta a algún estudiante, en el primer nivel de intervención estarían los docentes, actuando la jefa de UTP cuando la situación curricular se vuelve más compleja. Si otro es el caso, la misma autoridad puede decidir si la situación va "más alla’" y requiere otro tipo de intervención, es decir, cuando el tema no se supone propiamente "pedagógico" y se pasa a denominar "problema de convivencia".

Cabe destacar que el actor entrevistado da cuenta de una división entre pedagogía y convivencia, y de la construcción de un rol docente centrado en el rendimiento escolar, en el cual el abordaje de la convivencia no cabe, pues esa sería tarea de los profesionales de apoyo psicosocial (López, Morales \& Ayala, 2011). Esto es concordante con la noción de que habría ciertas situaciones que corresponden y/o están dentro de la capacidad de los docentes, habiendo otras que exceden lo que los docentes pueden y deben hacer. De esta manera, el establecimiento en vez de invitar a que toda la comunidad escolar se haga cargo, participe y se comprometa con la buena convivencia, podría estar burocratizando y delegando la tarea en 'especialistas' (Magendzo et al., 2013). Además, este escenario fomenta que el participante perciba un accionar delimitado por otras autoridades del establecimiento, lo cual es especialmente significativo para la estructuración del propio cargo de ECE:

Si yo fuera director le diría al ECE cuál es su trabajo y qué es lo que yo espero, estas son sus funciones, y buscaría socializarlo con los demás colegas, con los profesores sobre todo, para que sepan a quién tienen que dirigirse en caso de cualquier cosa (Tercera entrevista, ECE). 
En segundo lugar, se denota que aunque serían los actores del "área de la convivencia" los que se hacen cargo de lo "externo" a la pedagogía, la definición de "problema de convivencia” ya vendría dada por autoridades como la jefa de UTP y el director, definiendo los protocolos de acción ante las situaciones problemáticas que se observan en los estudiantes. Este aspecto termina fomentando la escisión que se produce entre el eje del aprendizaje y de la convivencia, como si se tratasen de dos asuntos con racionalidades distintas (Carrasco et al., 2012). Aquí surge un conflicto en la capacidad de toma de decisiones del ECE dentro del establecimiento, ya que su rol estaría dentro de una cadena de derivación donde la convivencia, y también la psicología, aparecen como la última opción, dotándolas del poder de transformar conductas (López et al., 2011): de allí que un primer paso para posicionar al ECE como un actor relevante en la convivencia escolar debe ser el empoderamiento del cargo.

Sin establecer este primer paso, pareciera que los actores de la convivencia están destinados a seguir reproduciendo el sistema jerárquico, o aún más, a quedar relegados en un área que está simbólicamente separada de los demás, y que por lo mismo establece que tanto las políticas de prevención, como los protocolos de actuación, sean considerados como algo diferente de las medidas pedagógicas (Carrasco, López \& Estay, 2012).

\section{Discusión}

Los resultados de la presente investigación permiten comprender cómo se ha ido construyendo la Identidad Laboral de este ECE en particular. A través del análisis realizado y teniendo como referencia teórica el concepto de identidad laboral (Garabito, 2005), hemos visualizado que el quehacer del ECE se guía por su interpretación personal y las valoraciones culturales que establece del trabajo institucional, las que son identificadas a través de su discurso y están marcadas por la diferenciación de las áreas administrativa, pedagógica y convivencia; 
sus creencias respecto al cómo se debe trabajar; y la concepción de un perfil de cargo ideal. Estas valoraciones se encuentran limitadas por su interpretación en torno a las estructuras sociales en las cuales se desenvuelve, lo que genera en él la identificación de dificultades debido a lo ambiguo que le resulta el área de convivencia escolar, y las funciones que debe ejercer como Inspector-ECE. Esto trae consigo una sensación de sobrecarga e incompatibilidad de horarios entre los cargos que desempeña en el establecimiento, lo cual se suma a la presión subjetiva que siente en un contexto de control, potenciado por la posibilidad de sanción de organismos ministeriales como la Superintendencia de Educación. Y es que, como describen Carrasco et al. (2012), a pesar de la creación de un cargo cuya función es abordar la convivencia escolar, este es creado en un estructura normativa centrada en el control y la vigilancia, y no en la relevancia al aspecto formativo.

Así, los resultados sugieren que la identidad del ECE tiene un carácter ambiguo. Esto guarda relación con la coexistencia de dos paradigmas en la propia ley que creó el cargo de ECE (Carrasco et al., 2012; Magendzo et al., 2013). Como hemos señalado, la ambigüedad prescriptiva hace suponer que se podrían adoptar distintas posiciones como ECE. En este caso estudiado, el ECE separa sus funciones de inspector, con las de ECE. El efecto de esta práctica discursiva es segmentar para diferenciar y separar, en este caso, la convivencia escolar de la pedagogía. Más aún, la diferenciación entre pedagogía y convivencia escolar aparece como estrategia para expresar las supuestas divisiones entre docentes y actores de la convivencia. El discurso del entrevistado da cuenta de las prácticas de individualización y externalización que derivan de concepciones sobre el rol docente que tienden a separar el "rendimiento" de la "convivencia y manejo de conflictos" (López et al., 2011).

Dado este escenario, donde también se incluye la noción de que el participante tiene que dedicar gran parte de su tiempo a labores administrativas, concluimos que la identidad laboral del ECE se presenta como un campo demarcado por la diferenciación entre las áreas de la pedagogía, la administración y la convivencia. Esto sería un reflejo 
de su trayectoria laboral donde ha ejercido labores como docente de inglés, inspector general, y posteriormente ECE.

Se puede establecer que un primer elemento poco definido es la labor de inspector, ya que el término "inspeccionar" se torna muy amplio en la práctica, pudiendo abarcar tanto la mantención de la escuela como la vigilancia de otros actores educativos para evitar errores. En el área de la pedagogía, pareciera que la situación es contraria porque se buscaría delimitar el proceso de aprendizaje a un campo curricular que deja fuera el aprendizaje en convivencia, aún cuando la convivencia escolar se debiera identificar, planificar y evaluar como cualquier otro aprendizaje y/o contenido, donde se enseña y se aprende a convivir (Mineduc, 2013).

Es así que hablar de una identidad laboral ambigua del ECE no aparece algo extrańo, ya que da cuenta de cómo su experiencia laboral hace relación con las tensiones y contradicciones que el sujeto presencia en su medio educativo. Es relevante generar un contexto educativo donde sus actores puedan percibir mayor claridad, horizontalidad y coordinación entre las áreas de administración, pedagogía y convivencia escolar. Hasta qué punto esta división es coherente es algo que es necesario cuestionar, considerando las posibilidades como el fortalecimiento de estructuras de toma de decisiones con representación de la comunidad educativa, como el Consejo Escolar o Comité de Buena Convivencia, buscando potenciar la organización colectiva entre padres, profesores y estudiantes alrededor de un Plan de Gestión (Carrasco et al., 2012).

El ECE de este estudio buscó identificar en este plano posibilidades y soluciones ante la presión que experimenta. Sin embargo, estas se presentan como difíciles de llevar a la práctica en un corto plazo, y sometidas al designio de otras autoridades. Estas serían la creación de un cargo administrativo que aliviaría, idealmente, tanto su sobrecarga laboral como su incompatibilidad de horario, así como la reestructuración que debería experimentar el área de convivencia escolar en relación a otras "áreas" de la escuela. Esta tensión a nivel intersubjetivo presenta una de las contradicciones más evidentes del discurso del entrevistado, que por un lado manifiesta una sensación de incompatibilidad respecto 
a los cargos que ejerce dentro del establecimiento como InspectorECE, realizando por otro lado una connotación positiva de la unión entre lo punitivo y lo formativo como condición ideal para ejercer un buen trabajo como ECE.

Los resultados obtenidos podrían ser congruentes con la experiencia de otros encargados de convivencia escolar en contextos similares. No obstante, una clara limitación de este estudio es su carácter de caso único de tipo exploratorio, que no permite la generalización. Además, ante la falta de estudios previos sobre el tema, dado que el cargo de ECE es relativamente nuevo y único, se vuelve relevante seguir investigando para dar cuenta de la identidad laboral en otros contextos educativos, donde actores con otras formaciones profesionales y otras designaciones de labores ocupen este cargo. Desde este punto de vista, sería interesante indagar en la experiencia de quienes están siendo llamados a abordar la convivencia escolar desde leyes creadas en otros países, como por ejemplo en Perú.

Esto responde a una necesidad de refrescar la narrativa en torno a la concepción tradicional de la convivencia escolar, que la entiende tan solo como una aspiración instrumental ya sea para mejorar el rendimiento académico o para reducir la violencia en la escuela. La nueva narrativa en cambio debe centrarse en la aspiración legítima de mejorar los procesos democráticos, justicia social y bienestar subjetivo al interior de la escuela (López, Díaz \& Carrasco, 2015). Por esto se hace necesario ir más allá de lo que no se debe hacer, como ha sido el caso de la mayoría de las políticas públicas en torno al tema, para empezar a fomentar procesos con el objetivo de que haya una coherencia entre lo que se enseńa y se practica al interior de la escuela.

\section{Referencias}

Arístegui, R., Bazán, D., Leiva, J., López, R., Muñoz, B. \& Ruz, J. (2005). Hacia una Pedagogía de la Convivencia. Psykhe, 14(1) 137-150. https://doi.org/10.4067/s0718-22282005000100011 
El encargado de convivencia escolar en Chile / Valenzuela et al.

Cáceres, P. (2003). Análisis cualitativo de contenido: Una alternativa metodológica alcanzable. Psicoperspectivas, 2, 53-82. Recuperado de http://www.psicoperspectivas.cl/index.php/ psicoperspectivas/article/viewFile/3/3.

Carrasco, C., López, V. \& Estay, C. (2012). Análisis crítico de la ley de violencia escolar de Chile. Psicoperspectivas, 11(2), 31-55. https:// doi.org/10.5027/psicoperspectivas-vol11-issue2-fulltext-228

Chadwick, A., Allamand, A. \& Cantero, C. (2010). Moción de los honorables senadores Chadwick, Allamand y Cantero, con la que inician un proyecto de ley sobre violencia escolar (Moción parlamentaria en sesión 41, legislatura 358). Recuperado de la página web de la Biblioteca del Congreso Nacional de Chile http://www.bcn. $\mathrm{cl} /$ historiadelaley/nc/historia-de-la-ley/4600/?

Collazos, H. (2007). Técnicas de Investigación. Bogotá, UNAD. Recuperado de http://www.emagister.com/uploads_courses/ Comunidad_Emagister_59486_investigacionfinal.pdf

Erickson, E. (1977). La identidad psicosocial. En D.L. Sills (Ed.), Enciclopedia Internacional de las Ciencias Sociales (Vol. 5., pp. 586-591). Madrid: Aguilar.

Fernández, M. \& Fernández, M. (2012). Aprendiendo a vivir juntos para vivir bien: Propuesta de procedimientos para el funcionamiento interno de Unidades Educativas. Manual de Funciones y Reglamento para la Convivencia Escolar con enfoque de Cultura de Paz. La Paz: Programa de Apoyo a la Gestión Pública Descentralizada Y Lucha contra la Pobreza de la Cooperación Técnica Alemana GIZ/PADEP. Recuperado de http://www.bivica.org/ upload/convivencia-escolar.pdf

Garabito, G. (2005). El trabajo en la identidad y la identidad en el trabajo. Trabajo presentado en el Encuentro Regional de la Asociación Mexicana de Estudios del Trabajo A.C., Querétaro. Recuperado de https://www.google.cl/url?sa=t\&rct=j\&q=\&esr$\mathrm{c}=\mathrm{s} \&$ source $=$ web\&cd $=1 \& \mathrm{cad}=$ rja\&uact $=8 \& \mathrm{ved}=0 \mathrm{ahUKEwi} 0$ m62U0JTQAhUBvZAKHUX-BecQFggZMAA\&url=http\%3 $\mathrm{A} \% 2 \mathrm{~F} \% 2 \mathrm{~F} w w w . i z t a p a l a p a . u a m . m x \% 2 \mathrm{Famet} \% 2 \mathrm{~F}$ congresoque 
retaro\%2Fponencias\%2F4_trabajo_la\%2520identidad.doc\&us $\mathrm{g}=$ AFQjCNFUGSq41 tBaegRQ6JuUaKagnCEmNA\&bvm=bv. 137904068 ,d.Y2I

Gobierno de Chile (2006). Estudio Nacional de Violencia en el ámbito Escolar (Informe ejecutivo). Santiago, Ministerio del Interior y Ministerio de Educación. Recuperado de http://www.seguridadpublica.gov.cl/filesapp/pres_ppt.pdf

Korman, A. (2003). Psicología de la industria y de las organizaciones. Madrid: Marova.

Ley establece la Ley General de Educación. Ley 20.370. Promulgada 17 agosto 2009. Ministerio de Educación, Gobierno de Chile. Recuperado de https://www.leychile.cl/Navegar?idNorma=1006043

Ley sobre violencia escolar (LSVE). Ley 20.536. Promulgada 8 de septiembre de 2011. Ministerio de Educación, Gobierno de Chile.

López, M. \& García, R. (2011). Convivir en la escuela: Una propuesta para el aprendizaje por competencias. Revista de Educación de la Universidad de Valencia, 356, 531-555. doi: 10-4438/1988-592X-RE-2010-356-050

López, V., Morales, M. \& Ayala, A. (2011). El encapsulamiento de los psicólogos escolares y profesionales de apoyo psicosocial en la escuela. Revista Internacional Magisterio, 53, (Número especial Bullying), 54-57.

López, V., Carrasco, C., Morales, M., Ayala, A., López, J. \& Karmy, M. (2011). Individualizando la violencia escolar: Análisis de prácticas discursivas en una escuela municipal de la Región de Valparaíso. Psykhé, 20 (2), 75-91. http://dx.doi.org/10.4067/ S0718-22282011000200002

López, V., Díaz, H. \& Carrasco, C. (Eds.) (2015). Nosotros si podemos: Aprendiendo a mejorar la convivencia escolar. Valparaíso: Pontificia Universidad Católica de Valparaíso y Centro de Investigación Avanzada en Educación (CIAE).

Magendzo, A., Toledo M. \& Gutiérrez, V. (2013). Descripción y análisis de la Ley sobre Violencia Escolar (No 20.536): Dos 
paradigmas antagónicos. Estudios Pedagógicos 39(1), 377-391. https://doi.org/10.4067/s0718-07052013000100022

Marshall, C. \& Rossman, G. B. (1989). Designing qualitative research. Newbury Park, CA: Sage.

Ministerio de Educación (Minedu). (2012). Ética, Democracia y Gobernabilidad desde la convivencia escolar: Tutoría y Orientación Educativa. Lima, Perú: Gobierno de Perú. Recuperado de http://www.unodc.org/documents/peruandecuador/ Publicaciones/etd_esc.pdf

Ministerio de Educación (Minedubo). (2012). Gestión y planificación educativa. La Paz, Bolivia: Gobierno de Bolivia. Recuperado de http://www.minedu.gob.bo/files/publicaciones/biblioteca/ gestion_y_planificacion_educativa.pdf

Ministerio de Educación (Mineduc). (2013). Gestión de la buena convivencia: Orientaciones para el encargado de convivencia escolar y equipos de liderazgo. Santiago, Chile: Gobierno de Chile. Recuperado de http://www.mineduc.cl/usuarios/convivencia_escolar/ doc/201309091630460.GestiondelaBuenaConvivencia.pdf

Mejía, N. (2000). El Muestreo en la investigación cualitativa. Revista del instituto de Investigaciones Histórico Sociales, 4(5), 165-180.

Mella, O. (2000). Grupos focales: Técnicas de Investigación Cualitativa. Santiago, Chile: CIDE.

Morales, J. (1999). La identidad social. Anthropológica: Revista de Etnopsicologia y Etnopsiquiatría, (17), 47-69.

Organización de las Naciones Unidas para la Educación, la Ciencia y la Cultura. (2007). Educación de calidad para todos: Un asunto de derechos humanos. Santiago, Chile: Autor, Oficina Regional de Educación de la Unesco para América Latina y el Caribe.

Organización de las Naciones Unidas para la Educación, la Ciencia y la Cultura. (2008). Convivencia democrática, inclusión y cultura de paz: Lecciones desde la práctica educativa. Santiago, Chile: Autor, Oficina Regional de Educación de la Unesco para América Latina y el Caribe. 
Palomino, L. (2012). La convivencia en las Escuelas. En L. Benites, J. Carozzo, V. Horna, L. Palomino, C. Salgado, C. Uribe \& L. Zapata (Eds.), Bullying y convivencia en la escuela: Aspectos conceptuales, aplicativos y de investigación (pp. 37-74). Lima, Perú: Observatorio sobre Violencia y Convivencia en la Escuela.

Piqueras, A. (1996). La identidad valenciana: La difícil construcción de una identidad colectiva, Madrid, Espańa: Institució Valenciana D`estudios I Investigació.

Quintana, A. (2006). Metodología de Investigación Científica Cualitativa. En A. Quintana y W. Montgomery (Eds.), Psicología: Tópicos de actualidad (pp.47-84). Lima, Perú: Universidad Nacional Mayor de San Marcos. Recuperado de http://cienciassociales.webcindario.com/PDF/Cualitativa/Inv_quintana.pdf

Raczynski, D. \& Muñoz, G. (2005). Efectividad escolar y cambio educativo en condiciones de pobreza en Chile. Santiago, Chile: Maval Ltda.

Rivas, J. \& Leite, A. (2010). The empire strikes back: Rediscovering the technical logic in the European university system. En J. Paraskeva (Coord.), Unaccomplished Utopia: Neo conservative dismantling of public higher education in European Union (pp. 131-147). Boston: Sense Publisher.

Recibido: 18 de noviembre, 2016

Revisado: 2 de octubre, 2017 Aceptado: 16 de octubre, 2017 